\section{$\underset{\text { hommes }}{\text { \& migrations }}$}

\section{Hommes \& migrations}

Revue française de référence sur les dynamiques

migratoires

$1303 \mid 2013$

Diasporas marocaines

\title{
Les migrants au Parlement !
}

La revendication du droit de vote et d'éligibilité aux élections législatives marocaines

\section{Antoine Dumont}

\section{OpenEdition}

\section{Journals}

Édition électronique

URL : http://journals.openedition.org/hommesmigrations/2567

DOI : 10.4000/hommesmigrations.2567

ISSN : 2262-3353

Éditeur

Musée national de l'histoire de l'immigration

Édition imprimée

Date de publication : 1 juillet 2013

Pagination : 113-117

ISBN : 978-2-919040-23-0

ISSN : $1142-852 X$

Référence électronique

Antoine Dumont, « Les migrants au Parlement ! », Hommes \& migrations [En ligne], 1303 | 2013, mis en ligne le 31 décembre 2015, consulté le 03 mai 2019. URL : http://journals.openedition.org/ hommesmigrations/2567 ; DOI : 10.4000/hommesmigrations.2567 


\title{
LES MIGRANTS AU PARLEMENT! LA REVENDICATION DU DROIT DE VOTE ET D'ÉLIGIBILITÉ AUX ÉLECTIONS LÉGISLATIVES MAROCAINES
}

par ANTOINE DUMONT, professeur certifié d'histoire-géographie, académie de Nantes, docteur en géographie, membre associé de Migrinter (UMR 7301, CNRS / université de Poitiers)

\author{
La participation à la vie politique du Maroc est une revendication \\ essentielle pour les émigrés marocains. En jeu, la création \\ d'une véritable citoyenneté extraterritoriale. Au début \\ des années 2000, la mobilisation des associations de migrants \\ en France et en Europe a débouché sur la création \\ d'un Conseil de la communauté marocaine à l'étranger. \\ Si le combat de la représentation a été remporté, l'implication \\ effective des migrants dans la vie politique marocaine reste \\ encore en devenir.
}

L'émergence de la revendication électorale

Il y a dix ans, dans le dossier sur les "Marocains de France et d'Europe" d'Hommes \& Migrations, Marco Martiniello et Hassan Bousetta observaient la dynamique associative autour d'une nouvelle revendication : celle de voter et d'être candidat aux législatives marocaines du 27 septembre 2002. Ils notaient également qu'ayant échoué dans l'immédiat, cette mobilisation avait au moins ouvert la question de la représentation des Marocains de l'étranger. Cette dynamique était symptomatique, pour eux, du passage de la figure du "travailleur immigrê" à celle du "citoyen transnational". Depuis, la situation a évolué, au moins sur le plan institu- tionnel. Aujourd'hui, le recul semble suffisant pour comprendre pourquoi cette revendication a constitué une véritable rupture dans l'histoire migratoire marocaine. Cet article propose un bref retour sur cette mobilisation et ses suites observées de 2003 à 2007 auprès d'associations de migrants marocains en France, la plupart en région parisienne, toutes composées d'une majorité de migrants (récents ou non) et orientées vers le Maroc en tant qu'État, espace ou société ${ }^{1}$.

Longtemps les migrants marocains se sont tenus éloignés du champ électoral de leur pays d'origine. Si l'opposition au régime d'Hassan II (1958-1999) 
a été vivace au sein des communautés émigrées et, en particulier, dans les milieux étudiants et exilés, elle ne s'y exprimait pas en termes électoraux. Et pour cause : durant cette période, les partis d'opposition étaient strictement encadrés, sinon réprimés, et donc privés d'assises électorales, les scrutins eux-mêmes étant fort peu démocratiques. L'enjeu, pour ces opposants, était donc de dénoncer le caractère truqué des élections. Dans ce domaine, les militants les plus actifs furent ceux de l'Association des Marocains de France (AMF). Par exemple, lorsque, en 1984 les législatives succédèrent aux émeutes de janvier, l'AMF dénonça la "participation à de fallacieuses élections législatives boycottées par la majorité de l'immigration marocaine ${ }^{2 "}$. Cela n'empêchait nullement l'AMF et ses homologues en Europe d'être étroitement liés à des partis (pour l'AMF, l'UNFP puis l'USFP). De leur côté, les amicales étaient les instruments de l'État marocain et des partis gouvernementaux. En Europe, c'est en fait l'ensemble du champ associatif marocain qui constituait, jusque dans les années 1980, une excroissance spatiale du champ politique
Un Marocain qui acquiert la nationalité française reste marocain aux yeux de l'État d'origine. Celui-ci défend donc une conception extraterritoriale et inaliénable de la nationalité, dont il facilite la transmission. marocain, une "exopolitie $e^{3 "}$, selon l'expression de Stéphane Dufoix. Dans les années 1990, les revendications des associations de migrants envers leur État d'origine pouvaient toucher à la liberté de circuler, au droit de la famille, à l'investissement au pays ou à un retour digne ; mais revendiquer un droit de vote et d'éligibilité est une idée plus récente. L'alternance gouvernementale de 1998 a sans conteste joué un rôle. Mais c'est la diversification de la population migrante marocaine qui semble avoir été, sur le temps long, lélément décisif. Sans revenir sur l'évolution des flux et des stocks, rappelons qu'ils sont marqués par la dispersion géographique, la féminisation et le vieil- lissement, ainsi que par la mobilité sociale et économique, malgré les discriminations. L'essor des associations de développement, dont Thomas Lacroix traite dans ce numéro, s'explique en grande partie par cette diversification. En retour, leurs militants se trouvent confrontés à une série d'obstacles administratifs et techniques qu'ils imputent largement à l'état du système politique marocain. Enfin, cette revendication émerge dans un moment où les transferts financiers des émigrés atteignent des niveaux records, ce qui va bien entendu être un argument en sa faveur. L'autre argument majeur est juridique.

\section{Le fondement juridique d'une citoyenneté extraterritoriale}

Un Marocain qui acquiert la nationalité française reste marocain aux yeux de l'État d'origine. Celuici défend donc une conception extraterritoriale et inaliénable ${ }^{4}$ de la nationalité, dont il facilite la transmission ${ }^{5}$, mais qui est contradictoire avec l'exclusion, en pratique, des Marocains de l'étranger de l'exercice du droit de vote et d'éligibilité au Maroc. La plupart des acteurs mobilisés soulignent cette contradiction. Pourtant, ce droit a existé en 1984 pour élire la Chambre des conseillers. L'espace migratoire d'alors fut divisé en cinq vastes circonscriptions $^{6}$ et tout Marocain enregistré au consulat put être électeur et candidat. Les amicales s'engagèrent pleinement dans une campagne récusée par les associations d'opposants. Cette expérience, d'emblée critiquée, fut stoppée au prétexte de la création, en 1990, d'un ministre délégué en charge des Marocains de l'étranger, en place jusqu'en 1997. D'autres arguments contre l'exercice de ce droit apparurent ensuite, tous récusables sur le plan juridique ${ }^{7}$. Parmi d'autres, on citera l'article 41 de la Convention internationale, signée par

2. AMF, XII' congrès de l'AMF. Plateforme, programme revendicatif, motions, 1986. 3. Stéphane Dufoix, Politiques d'exil. Hongrois, Polonais et Tchécoslovaques en France après 1945, Paris, PUF, 2002. 4. À l'exception de certaines condamnations et des Marocains ayant acquis la nationalité d'un État n'autorisant pas la double nationalité. 5. Depuis 2007, le nouveau Code de la nationalité dispose que "tout enfant né de père marocain ou de mère marocaine est de nationalité marocaine" (art. 6). Ce droit est conditionné au caractère musulman du mariage et, en cas de naissance hors mariage, à la religion musulmane du père. $\mathbf{6}$. Le nord de la France et la région parisienne (Paris), le sud de la France (Lyon), l'Europe centrale et septentrionale (Bruxelles), les pays arabes (Tunis) et le reste du monde (Madrid), regroupant Grande-Bretagne, Italie et Espagne. 7. Abdelkrim Belguendouz, Marocains des ailleurs et Marocains de l'intérieur, Rabat, Beni Snassen, 2003. 
le Maroc, pour la protection des droits des travailleurs migrants, entrée en vigueur en 2002 : "Les travailleurs migrants et les membres de leur famille ont le droit de prendre part aux affaires publiques de leur État d'origine, de voter et d'être élus au cours d'élections organisées par cet État." Le fondement juridique aurait d'ailleurs pu être discuté par la Cour suprême, saisie par une coordination d'associations de migrants, mais la demande fut déclarée irrecevable (arrêt du 31 juillet 2003). En réalité, la situation marocaine n'est pas exceptionnelle. Si près de la moitié des États du monde reconnaissent la double citoyenneté ou la double nationalité ${ }^{8}$, la plupart des États de forte émigration ne permettent pas le vote à distance, à l'exception notable de l'Algérie et de la Tunisie. Le droit de vote et d'éligibilité implique généralement la domiciliation du national sur le territoire de l'État en question. La citoyenneté extraterritoriale se limite souvent, comme dans le cas du Maroc, à la protection diplomatique, au droit au retour et à d'autres droits civils et sociaux (droit à la propriété foncière, à la retraite, etc.). Même lorsqu'il existe, le droit de vote n'est pas toujours exercé : par exemple, une minorité de Portugais de létranger sont inscrits sur les listes consulaires et, aux élections législatives de 2002, l'abstention s'est élevée à $75 \%$ de ces 162612 inscrits $^{9}$. Sans lui dénier sa légitimité, il faut donc se demander si cette revendication répond à une demande sociale.

\section{La lutte pour la représentation}

En 2002, la mobilisation prend de l'ampleur. Les anciennes associations d'opposants et celles qui en sont issues joignent leur voix à celle des anciennes amicales. Ainsi, tandis que l'Association des travailleurs immigrés marocains d'Espagne dénonce, en juin 2002, "latteinte à la Constitution" que représenterait "lexclusion de 3 millions de Marocains immigrés aux élections de septembre", la Fédération des associations des Marocains d'Europe soutient cette revendication "dans le sens de la construction d'une nation marocaine plus forte et plus présente sur la scène internationale". Outre ces acteurs historiques du champ associatif, des groupes ad hoc se forment sur cette question et produisent quantité de textes (lettres ouvertes, manifestes, pétitions...) dont certains trouvent un large écho dans la presse marocaine ${ }^{10}$. Des individus se distinguent par la virulence de leurs propos, menaçant de créer un parti de l'immigration. Les associations de défense des droits de l'homme soutiennent également cette revendication et l'intègrent dans leur rapport commun sur les législatives de 2002, en la reliant à la démocratisation du pays. Dans le sillage de cette mobilisation En réalité, la situation marocaine n'est pas exceptionnelle. Si près de la moitié des États du monde reconnaissent la double citoyenneté ou la double nationalité, la plupart des États de forte émigration ne permettent pas le vote à distance, à l'exception notable de l’Algérie et de la Tunisie. apparaît une tentative de conseil représentatif des migrants, dont les membres seraient élus par les migrants.

En 2003, une petite centaine d'associations rejoint le processus. La participation est très affaiblie ensuite par des accusations de "communautarisme" et de trop grande proximité avec l'État marocain ou, plus simplement, par des difficultés pratiques. Au final, le 21 novembre 2004, une poignée d'associations organisent les élections, où 2200 Marocains désignent 74 "conseillers". Seuls 6 départements ont des représentants élus par plus d'une centaine d'électeurs : l'Essonne et les Yvelines (île-de-France), l'Yonne (Bourgogne), le Doubs (Franche-Comté), les Alpes-Maritimes et le Var (Provence-Alpes-Côte d'Azur) (voir cartes 1 et 2). Ce Conseil national des Marocains de France disparaîtra lorsque son président sera nommé au Conseil de la communauté marocaine à l'étranger (CCME) en 2007. D'autres trajectoires de ce type existent et les ambitions personnelles s'expriment clairement dans cette mobilisation. Cela nuit à sa réussite et à son caractère fédérateur. Ainsi, les jeunes nés en France, engagés dans des actions de développement du Maroc, pays de leurs parents, trouvent difficilement leur place dans ce débat sur 
réforme constitutionnelle visait d'abord à satisfaire les revendications sociales et politiques du mouvement dit du "20 février", le nouvel article 17 affirme que "les Marocains résidant à létranger jouissent des droits de pleine citoyenneté, y compris le droit d'être électeurs et éligibles". Toutefois, la loi devra fixer les conditions de son "exercice effectif (...) à partir des pays de résidence". Aux législatives anticipées de 2011, la candidature des migrants est donc impossible et seul un vote par procuration est mis en place, en vertu de l'article 69 du nouveau Code électoral. Les acteurs mobilisés protestent, sans résultat jusqu'à aujourd'hui.

\section{La création du Conseil de la communauté marocaine à l'étranger}

À l'inverse, l'autre promesse royale de 2005 s'est vue promptement réalisée.

Hanane, 32 ans, journaliste française, a écrit un livre sur la double culture @ SOPHIE PASOUET

Un décret de 2007 a créé le Conseil de la communauté marocaine à l'étranger, dont le droit de vote et d'éligibilité à distance. Et si d’anciens clivages s'estompent, tel celui entre "progressistes" et "amicalistes", l'État marocain reste bien présent dans la vie associative de "ses" migrants. Depuis 2002, trois temps peuvent être distingués dans la réponse de l'État marocain. Le premier est le discours du roi du 6 novembre 2005 qui annonce l'octroi prochain de ce droit tant réclamé et la création d'un Conseil des Marocains de l'étranger. Cette annonce a un écho considérable au Maroc et en France. Mais, le 16 juin 2006, le ministre de l'Intérieur marocain annonce que ce droit de voter et de se présenter à distance est inapplicable pour des “raisons techniques". En 2007 comme en 2002, ceux qui souhaitent voter devront rentrer. Le troisième temps est celui de la nouvelle Constitution, approuvée en juillet 2011 par un référendum ; les migrants ont pu, comme en 1996, voter à distance. Si cette les membres nommés sont chargés d'émettre des avis consultatifs. La nouvelle Constitution rappelle, dans son article 163, ses trois missions : maintenir l'identité marocaine des migrants, garantir leurs droits et favoriser leur contribution au développement du Maroc. La genèse du CCME, dont le président est interviewé dans ce numéro, entretient des proximités avec la mobilisation étudiée. En effet, l'idée d'un tel conseil est née dans les années 1990 au sein de l'immigration marocaine. Elle devient ensuite l'objet principal d'une association, le Congress, créée à Bruxelles en 2001 dans le sillage du Forum des migrants. Cette idée rencontre un écho favorable auprès de la ministre déléguée chargée des Marocains résidant à létranger (depuis 2003) et elle est au centre d'une campagne d'information au Maroc et en France de 2004 à 2005. Ce lobbying intense repose sur un 
fort sentiment patriotique, la relation des migrants au Maroc étant comparée à celle d'un enfant à ses parents ${ }^{11}$. Surtout, le Congress préfère que ce conseil soit composé de membres nommés plutôt quélus par les migrants, ce qui serait trop compliqué à appliquer. Cet argumentaire sera repris par le gouvernement marocain. Mais le Congress évoque aussi des risques de manipulation électorale "à cause de faiblesses liées à notre culture politique et sociale (clientélisme, favoritisme, manipulation, etc.)". Ce point de vue dénote franchement à une époque où la revendication du droit de vote et d'éligibilité gagne l'ensemble du champ associatif immigré marocain. Il n'est pourtant pas éloigné de la position de la ministre des Marocains de l'étranger qui, tout en légitimant cette revendication, semble poser comme préalable la "structuration de la communauté" au sein d'un conseil.

À l'inverse, la naissance du CCME semble contrarier les objectifs d'autres associations issues du pôle progressiste. Engagées dans la campagne pour le droit de vote, elles souhaitent aussi être consultées en tant qu'associations de migrants lors d'une conférence nationale sur l'immigration, dont l'idée vient du rapport de 2005 de l'instance Équité et réconciliation. La tentative de structurer à nouveau ce pôle progressiste est d'abord menée par le Centre euro-méditerranéen migration et développement (Emcemo) d'Amsterdam, puis par l'Association des travailleurs maghrébins de France (ATMF) et aboutit à la création du collectif Al Monadara fin 2006, où se retrouvent quelques dizaines d'associations d'Europe. L'ATMF réclame alors que les associations de migrants soient majoritaires au futur $\mathrm{CCME}^{12}$. Dans le même temps, un responsable d'Emcemo dénonce l'attention trop grande accordée aux élections législatives de 2007 et au futur $\mathrm{CCME}^{13}$. Le risque est pour lui de confondre vie associative et campagne partisane. Aujourd'hui, le CCME présente un bilan contrasté $^{14}$ et affirme son opposition au droit de vote et d'éligibilité des Marocains de l'étranger ${ }^{15}$. Cette mobilisation s'est déroulée selon de nouvelles lignes de clivage qui ne reproduisent pas l'opposition historique entre progressistes et amicalistes. Le débat semble aujourd'hui opposer les organes consultatifs, de type CCME, et les différentes formes de réseaux, collectifs et autres initiatives ad hoc qui réunissent d'anciens amicalistes, d'anciens gauchistes, des syndicalistes, des jeunes engagés dans le développement ou les activités culturelles et religieuses. Parmi eux, les professions intermédiaires et supérieures semblent surreprésentées. Les groupes mobilisés se définissent moins politiquement que socialement et restent des ensembles hétérogènes. Les liens de ces militants se sont renforcés des deux côtés, avec la France et le Maroc. Toutefois, on peut douter de l'enracinement de leurs organisations au sein de la population immigrée, notamment la plus récente. Revendiquer de voter "au pays" est une préoccupation marginale dans la vie quotidienne des immigrés. Enfin, leur approche dominante vis-à-vis de l'État marocain semble avoir changé : elle n'est plus fondée sur la méfiance et la confrontation mais sur le consensus et l'accommodation. En cela, elle s'inspire beaucoup de celle qui prévaut dans les associations de développement. ${ }^{16}$

La situation marocaine est finalement représentative d'un état de ce champ migratoire, dans lequel l'installation durable et les attaches nouées en Europe n'empêchent pas mais, au contraire, facilitent des liens plus étroits et apaisés avec la société d'origine. Elle montre que la citoyenneté extraterritoriale ou transnationale peut aller de pair avec une citoyenneté de résidence. Ce faisant, elle pose aussi la question de la légitimité des migrants à être des acteurs politiques dans deux espaces distincts. 